\title{
TAIGA - a hybrid array for high energy gamma astronomy and cosmic ray physics
}

$N$. Budnev ${ }^{2, *}, I$. Astapov ${ }^{9}, P$. Bezyazeekov $^{2}, V$. Boreyko $^{10}, A$. Borodin $^{10}, M$. Brueckner $^{8}$,

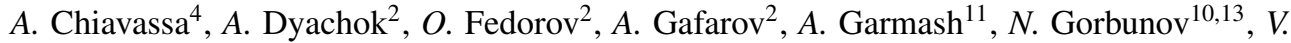
Grebenyuk $^{10,13}, O$. Gress ${ }^{2}, T$. Gress ${ }^{2}, O$. Grishin ${ }^{2}, A$. Grinyuk ${ }^{10}, A$. Haungs ${ }^{14}, R$. Hiller ${ }^{14}, D$. Horns $^{6}, T$. Huege ${ }^{14}, N$. Kalmykov ${ }^{1}, Y$. Kazarina ${ }^{2}, V$. Kindin $^{9}, S . K_{\text {Kiryuhin }}^{2}, P$. Kirilenko ${ }^{11}$, $M$. Kleifges ${ }^{15}, R$. Kokoulin ${ }^{9}, K$. Kompaniets ${ }^{9}, E$. Korosteleva ${ }^{1}, D$. Kostunin ${ }^{14}, V$. Kozhin ${ }^{1}$, E. Kravchenko ${ }^{11,12}, L$. Kuzmichev ${ }^{1}, Y u$. Lemeshev ${ }^{1}, V$. Lenok $^{14}, B$. Lubsandorzhiev $^{3}, N$. Lubsandorzhiev $^{1}, R$. Mirgazov ${ }^{2}, R$. Mirzoyan ${ }^{5}, R$. Monkhoev ${ }^{2}, E$. Osipova ${ }^{1}, A$. Pakhorukov ${ }^{2}$, M. Panasyuk ${ }^{1}, L$. Pankov ${ }^{2}, A$. Petrukhin ${ }^{9}, V$. Poleschuk ${ }^{2}, M$. Popescu ${ }^{13}, E$. Popova ${ }^{1}, A$.

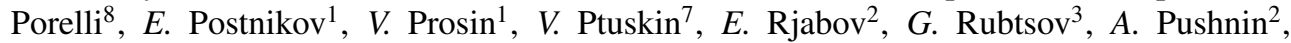
Y. Sagan ${ }^{10,13}$, B. Sabirov ${ }^{10}, V$. Samoliga ${ }^{2}, F$. Schröder ${ }^{14}$, Yu. Semeney ${ }^{2}$, A. Silaev ${ }^{1}, A$. Silaev (junior) ${ }^{1}, A$. Sidorenkov ${ }^{3}, A$. Skurikhin ${ }^{1}, V$. Slunecka ${ }^{10}, A$. Sokolov ${ }^{11,12}, C$. Spiering ${ }^{8}$, L. Sveshnikova ${ }^{1}, V$. Tabolenko ${ }^{2}, B$. Tarashansky ${ }^{2}, A$. Tkachenko ${ }^{10}, L$. Tkachev ${ }^{10,13}, M$. Tluczykont $^{6}, R$. Wischnewski ${ }^{8}, A$. Zagorodnikov $^{2}, D$. Zhurov $^{2}, V$. Zurbanov $^{2}$, and $I$. Yashin $^{9}$

${ }^{1}$ Skobeltsyn Institute of Nuclear Physics MSU, Moscow, Russia

${ }^{2}$ Institute of Applied Physics ISU, Irkutsk, Russia

${ }^{3}$ Dipartimento di Fisica Generale Universiteta di Torino and INFN, Torino, Italy

${ }^{4}$ Max-Planck-Institute for Physics, Munich, Germany

${ }^{5}$ Institut für Experimentalphysik, University of Hamburg, Germany

${ }^{6}$ IZMIRAN, Moscow, Russia

${ }^{7}$ DESY, Zeuthen, Germany

${ }^{8}$ National Research Nuclear University MEPhI, Moscow, Russia

${ }^{9}$ JINR, Dubna, Russia

${ }^{10}$ Novosibirsk State University, NSU, Novosibirsk, Russia

${ }^{11}$ Budker Institute of Nuclear Physics SB RAS, Novosibirsk, Russia

${ }^{12}$ ISS, Bucharest, Romania

${ }^{13}$ Dubna State University, Dubna, Russia

${ }^{14}$ Institut für Kernphysik, Karlsruhe Institute of Technology (KIT), Karlsruhe, 76021 Germany

${ }^{15}$ Institut für Prozessdatenverarbeitung und Elektronik, Karlsruhe Institute of Technology (KIT)

\begin{abstract}
The physics motivations and advantages of the new TAIGA (Tunka Advanced Instrument for cosmic ray physics and Gamma Astronomy) detector are presented. TAIGA aims at gamma-ray astronomy at energies from a few $\mathrm{TeV}$ to several $\mathrm{PeV}$, as well as cosmic ray physics from $100 \mathrm{TeV}$ to several $\mathrm{EeV}$. For the energy range $30-200 \mathrm{TeV}$ the sensitivity of $10 \mathrm{~km}^{2}$ area TAIGA array for the detection of local sources is expected to be $5 \times 10^{-14} \mathrm{erg} \mathrm{cm}^{-2} \mathrm{sec}^{-1}$ for $300 \mathrm{~h}$ of observations. Reconstruction of the given EAS energy, incoming direction and its core position, based on the timing TAIGA-HiSCORE data, allows one to increase a distance between the IACTs up to 600-1000 $\mathrm{m}$. The low investments together with the high sensitivity for energies $\geq 30-50 \mathrm{TeV}$ make this pioneering technique very attractive for exploring the galactic PeVatrons
\end{abstract}

*e-mail: nbudnev@api.isu.ru 
and cosmic rays. At present the TAIGA first stage has been constructed in Tunka valley, $50 \mathrm{~km}$ West from the Lake Baikal. The first experimental results of the TAIGA first stage are presented.

\section{Introduction}

A detection of gamma rays is one of the most efficient ways of investigating of galactic and metagalactic sources of high-energy cosmic rays as well solving of many problems of astroparticle physics. By the present time, the most significant results in ground based gamma astronomy have been obtained using the arrays HEGRA, H.E.S.S., VERITAS and MAGIC, which include from 2 to 5 Imaging Atmospheric Cherenkov Telescopes (IACT). The method proposed by Hillas is used for images analysis. To reliably reconstruct the EAS parameters and determine the nature of the original particle, EASs should be detected by several IACTs located at distance about $100 \mathrm{~m}$. The relatively small area of the currently operating arrays does not allow one to detect high energy photons with sufficient statistics. Arrays with an effective area at least one square kilometer are required to study the gamma rays with energy above $50 \mathrm{TeV}$. For this reason, the Cherenkov Telescope Array (CTA) should include Small Size Telescope (SST) subarray with about 70 IACT with diameter about $4 \mathrm{~m}$ on an area $7 \mathrm{~km}^{2}$. A conceptually new approach to the studies of high-energy gamma rays is being developed in TAIGA gamma observatory with a hybrid system of jointly functioning wide-angle timing and imaging Cherenkov detectors.

TAIGA is currently being constructed in the Tunka Valley, $50 \mathrm{~km}$ from Lake Baikal (Fig. 1). The main feature of TAIGA is the reconstruction of an energy, position, and direction of the EAS axis using the data of the wide-angle Cherenkov timing array TAIGA-HiSCORE (High Sensitivity COsmic Rays and gamma Explorer) [1]. Imaging Atmospheric Cherenkov Telescopes TAIGA-IACT will be used to select gamma rays from hadron background. Very important that for this purpose they can operate in the mono-mode and the distance between the IACTs can be increased at least up to $600 \mathrm{~m}$ and possibly up to $1000 \mathrm{~m}$. At present, the timing TAIGA-HiSCORE array on an area of $0.5 \mathrm{~km}^{2}$ about with 55 wide - angle Cherenkov detectors with the field of view (FOV) of $0.6 \mathrm{sr}$ and the first IACT of the TAIGA-IACT are in operation. Up to the end of $2019 y$, the number of wide-angle Cherenkov detectors of the TAIGA-HiSCORE array will be doubled, and two more IACTs of the TAIGA-IACT array will be put in operation. The deployment of muon detectors with a total area of $250 \mathrm{~m}^{2}$ is planned as well. Such a complex with an area of approximately one square kilometer will make it possible to experimentally test the efficiency of the joint operation of the wide-angle Cherenkov array, IACT, and muon detectors for detecting gamma rays with energy above 30 $\mathrm{TeV}$ and will also become the most sensitive instrument for solving the problems of highenergy gamma astronomy and make it possible to obtain the data on cosmic rays with energy above $100 \mathrm{TeV}$ with the record-setting level of accuracy and statistics. Simultaneously, for the purpose of studying the cosmic ray fluxes and searching for higher-energy gamma rays and neutrinos, a method is being actively developed that involves detecting the EAS radio emission using the Tunka-Rex array (the threshold energy of detection is $100 \mathrm{PeV}$ for hadronic EASs and $10 \mathrm{PeV}$ for gamma-ray-initiated electromagnetic EASs) that will operate jointly with the wide - angle timing Cherenkov Tunka-133 array as well as Tunka-Grande and TAIGA-Muon scintillation arrays. The rapid progress in the construction of the TAIGA gamma observatory is achieved through the use of technical experience accumulated during the development of a number of the wide-angle Cherenkov arrays (Tunka-13, Tunka-25 [2], and Tunka-133 [3]), as well as the Tunka-Rex radio array [4] and the Tunka-Grande scintillation array [5]. 


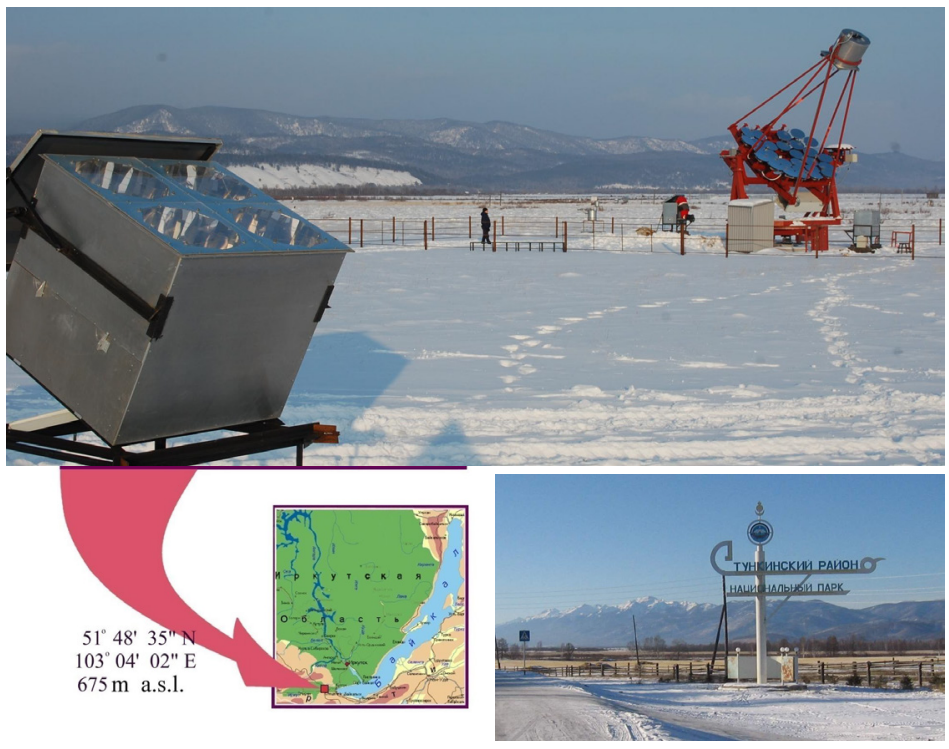

Figure 1. Wide-angle timing and imaging Cherenkov detectors of gamma observatory TAIGA in the Tunka Valley

\section{TAIGA-HiSCORE wide-angle Cherenkov array}

Each Cherenkov detector of the TAIGA-HiSCORE array includes two containers. In one of them (the Cherenkov container, Fig. 2) there are four photomultipliers (PMT) with a photocathode of 20 to $25 \mathrm{~cm}$ diameter (ET9352KB, R5912, or R7081). Each PMT is equipped with a Winston cone made of a high reflective material Alanod 4300 UD, which increases the effective light-collecting area by a factor of four. The FOV of the cone is about $0.6 \mathrm{sr}$. The light-collecting cones are covered with UV-transparent plexiglass for protection from dust, moisture and frost in winter. To protect the PMTs from sunlight and precipitation the Cherenkov containers are equipped with lids that open and close driven by electric motors. The data acquisition system of the TAIGA-HiSCORE array has a hierarchical structure. The Cherenkov detectors of the array are divided into clusters of approximately 30 detectors each.

The data acquisition system of each cluster includes two parts: the data acquisition electronics of the Cherenkov detectors (Detector DAQ), which is located in the thermally stabilized "electronic containers", and the central part (Central DAQ), located in the geometrical center of the cluster. Each Cherenkov detector is connected to the central DAQ of the cluster by an optical cable, which serves for the data transmission and synchronization. The synchronization is performed with a subnanosecond accuracy using the hybrid system. It combines the traditional 100-MHz synchronization system and the White Rabbit (WR) Ethernet-based time system [6], . The latter provides the reference to the central clock based on the GSPdisciplined Rubidium Oscillator. The stability of the synchronization is on the order of 0.2 ns. The main components of the Cherenkov detector DAQ are the analog summators and 8-channel ADC based on the DRS-4 board, by means of which the signals from the anodes and fifth dynodes of each PMT (for expanding the dynamic range) are digitized with a 0.5-ns step within a 200-ns window after the formation of the trigger. At a moonless night the trigger counting rate of a TAIGA-HiSCORE Cherenkov detector is about 10-15 Hz. The energy threshold of the array is $80-100 \mathrm{TeV}$ for detection of hadronic EASs and $40-50 \mathrm{TeV}$ for detec- 
tion of EAS initiated by gamma rays. During the winter season of 2017-2018, 43 Cherenkov detectors of the first and second clusters of the TAIGA-HiSCORE array participated in the data taking (Fig. 3). They are located on an area of $0.4 \mathrm{~km}^{2}$ with the spacing of $106 \mathrm{~m}$. All Cherenkov containers were tilted southward by 25 degrees to increase the time of observation of the gamma ray source in the Crab Nebula. The EAS parameters are reconstructed using the methods and algorithms developed for processing the data of the Tunka-133 array [9], [10]. The direction of the EAS arrival is determined from the relative time delays of the Cherenkov pulses by each detector. The accuracy of determining the direction of the EAS arrival greatly depends on the number of the detectors triggered. The angular resolution is 0.4-0.5 degree[7]s for the events with 4-5 detectors triggered and approximately 0.1 degrees for the events with more than 10 detectors triggered. The accuracy of the reconstruction of the EAS direction was tested by the detection of light from the lidar installed at the ISS [11]. A comparison of the reconstruction results of the ISS position from the TAIGA-HiSCORE array data and from the robotic telescope of the MASTER data located at the same site [12] shows the coincidence with the discrepancy less than 0.1 degree. The primary particle energy is reconstructed from the density of the Cherenkov light flux at $200 \mathrm{~m}$ distance from the EAS axis $Q_{200}$ with an accuracy of approximately $15 \%$ [10]. Figure 4 (left) shows the preliminary results of the reconstruction of the cosmic-ray energy spectrum from the TAIGA-HiSCORE array data in comparison with the results of our previous experiments in the Tunka Valley, as well as with the results of the ATIC-2 balloon experiment [13] and NUKLON satellite experiment [14]. Figure 4 (right) shows the results of the search the SN 1054 supernova remnant, or the Crab Nebula (further, the Crab) gamma emission in the energy range of 40-100 TeV in the cell of $0.3-0.4$ degrees in the direction toward the Crab. Preliminarily the number of the detected EAS exceeds the cosmic ray background at the confidence level of 2-2.5.
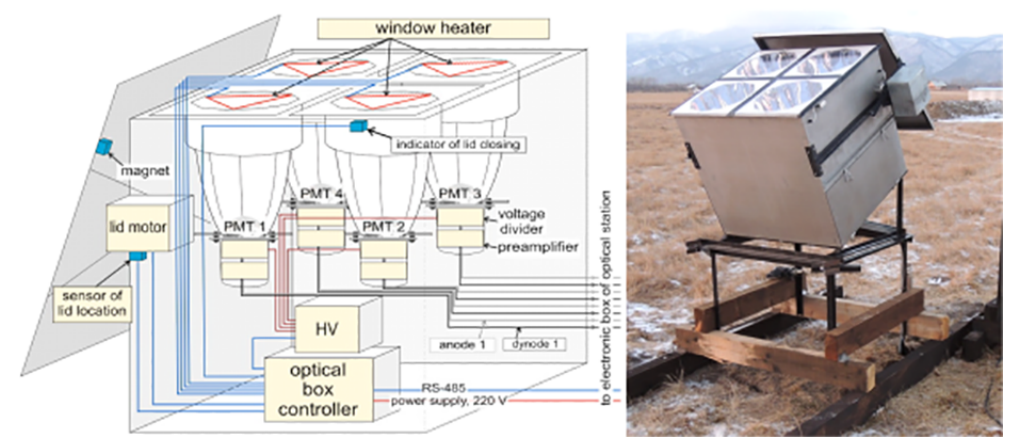

Figure 2. Schematic diagram of the Cherenkov container with the PMT of a Cherenkov detector of the TAIGA-HiSCORE array (left) and its photograph (right).

\section{THE TAIGA-IACT array}

The Imaging Atmospheric Cherenkov Telescopes of the TAIGA-IACT array have a Davis-Cotton mirror with the total area $10 \mathrm{~m}^{2}$ about and focal distance $4.75 \mathrm{~m}$. The telescope camera with a FOV of $9.7 \times 9.7$ degrees includes 547 photomultipliers XP1911. Each PMT is equipped with a Winston cone with an entrance size $300 \mathrm{~mm}$ and an exit spot of $15 \mathrm{~mm}$ diameter. The total diameter of the camera is $110 \mathrm{~cm}$ about. The camera PMTs are grouped into clusters, each cluster contains four groups of seven PMTs with a voltage divider board and high-voltage power supply. Each cluster equipped with a cross-board with a 


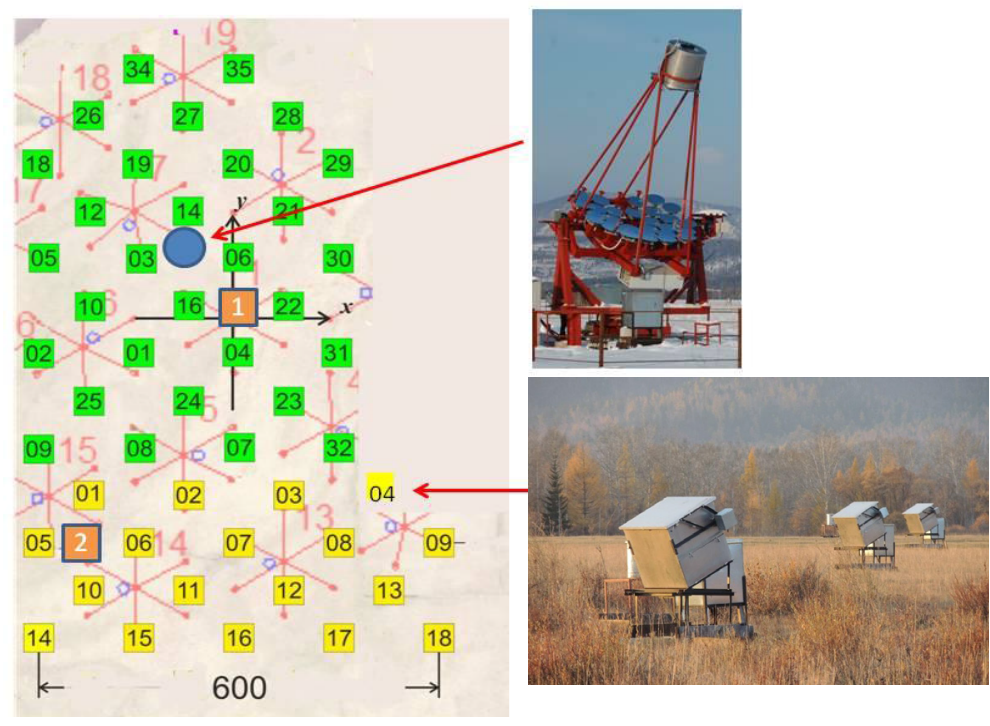

Figure 3. TAIGA-HiSCORE timing array layout since 2017. Cherenkov detectors are shown in green (first cluster) and yellow (second cluster) with the Tunka-133 cosmic ray array (red).Blue circle - first TAIGA-IACT.

DAC for controlling of the high-voltage sources and 12 bit ADC based on a 64-channel chip ASIC MAROC-3. During the winter season 2017-2018, more than 10 thousand joint events were detected by the TAIGA-HiSCORE and the first telescope of the TAIGA-IACT, including more than 300 events in the angle with a solution of 0.7 degrees relative to the direction of the Crab (during the measurement 10-20 EAS were expected, generated by gamma quanta with energies above $40 \mathrm{TeV}$ ). In Fig. 5 on the left there is an example of an EAS image, probably generated by a hadron, and on Fig. 5 (right) of an event generated by a gamma quantum.

\section{Scintillation and radio arrays as part of the TAIGA gamma observatory}

The most significant disadvantage of Cherenkov detectors with regard to the problems of cosmic ray physics and gamma astronomy is that they can operate only on moonless nights in good weather conditions, which reduces the time available for data taking almost by an order of magnitude. Scintillation particle detectors and radio detectors not only allow one the allday and all-weather observations, but also make it possible to solve several important physical problems; in particular, the data on the muon number are very useful for determining the type of the primary particle that initiated the EAS and especially for distinguishing the gamma rays, since the number of muons in a photon-initiated EAS less than in a proton-initiated EAS. Due to this fact, the Tunka-Grande array was constructed in 2013-2015, consisting of 19 scintillation stations on an area of $1 \mathrm{~km}^{2}$ [5]; the stations are based on the counters that previously were used as a part of the EAS-TOP and KASCADE-Grande arrays. Each station has surface part with 12 counters of total area $7.5 \mathrm{~m}^{2}$ and underground part with 8 counters of total area of approximately $5 \mathrm{~m}^{2}$. To reliably distinguish an EAS from gamma rays, the total area of muon detectors should be at least $0.2 \%$ of the area of the array. For this reason new 

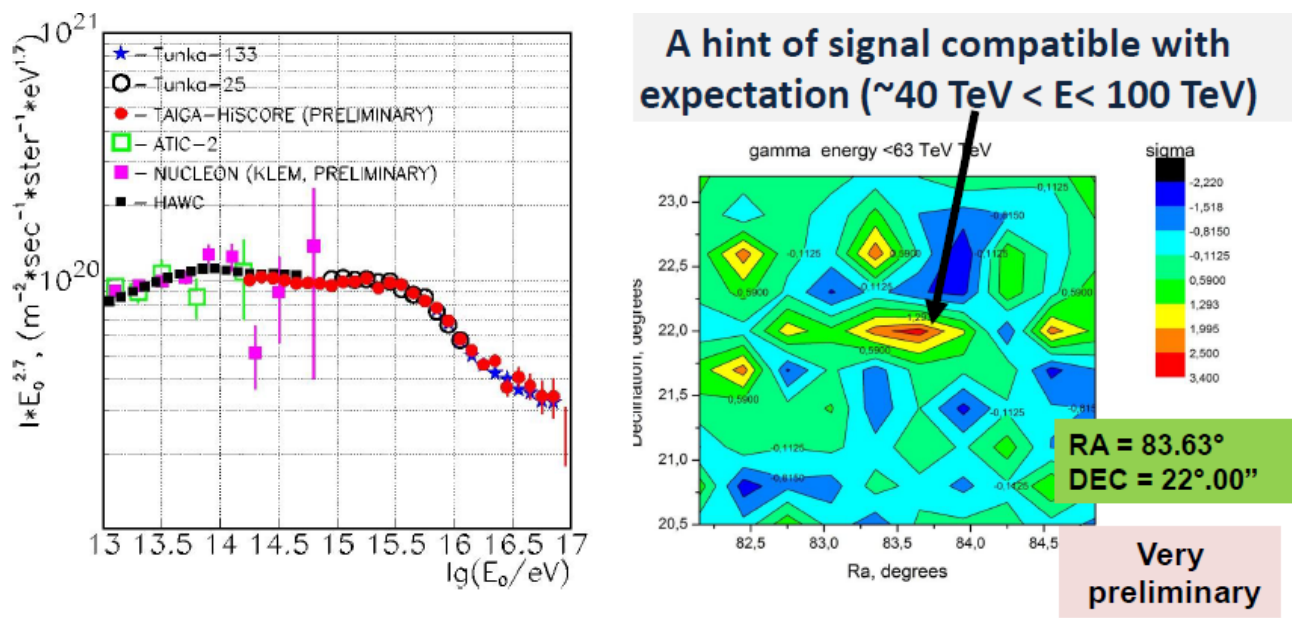

Figure 4. The energy spectrum of primary cosmic rays from the data of the TAIGA-HiSCORE array compared with the results of other experiments (left). The results of search for the EAS excess over the cosmic ray background in the energy range $40-100 \mathrm{TeV}$ in the cell of a $0.3-0.4$ degrees in the direction toward the Crab (right).
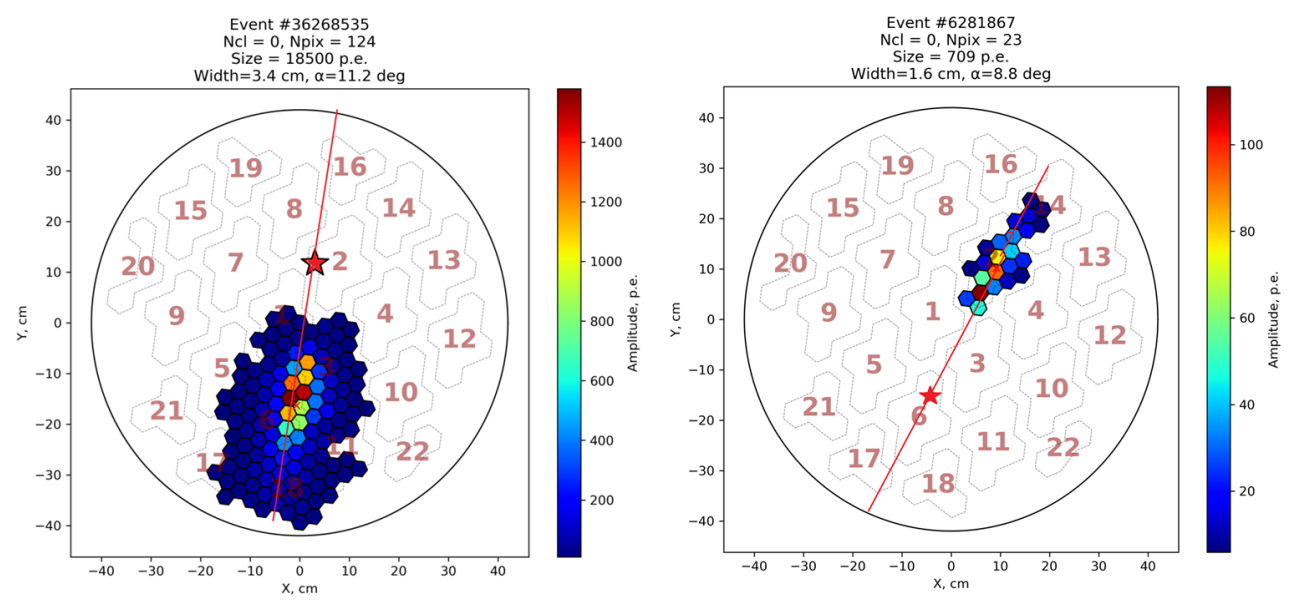

Figure 5. "Hadron-like" event (left), "gamma-like" event $\left(\mathrm{Gam}<1^{\circ}\right.$, width $\left.<0.13^{\circ}\right)$ (right). The star on both plots means the projection of the shower axis position, obtained from the data of the TAIGAHiSCORE array and recalculated into a coordinate system of the TAIGA-IACT camera.

scintillation counters have been developed and tested, they will become the basis of the new TAIGA-Muon array, where the total area of muon detectors will reach $2000-3000 \mathrm{~m}^{2}$. For the collection of the scintillation light and its transition to PMT wavelength shifting bars with a cross-section of $5 \times 20 \mathrm{~mm}$ are used.The mean amplitude for a particle passage through the counter is 23.1 photoelectrons; the nonuniformity is less than $15 \%$ (minimum-maximum). The time resolution of the counter is at least $5 \mathrm{~ns}$. 
At present the Tunka Radio Extension (Tunka-Rex) is an array of 63 antennas distributed on an area of $3 \mathrm{~km}^{2}$. Each Tunka-Rex antenna station consists of two perpendicular short aperiodic loaded loop antennas (SALLA). The effective band of Tunka-Rex is $30-80 \mathrm{MHz}$, it allows one to obtain maximum signal-to-noise ratio (SNR), and Earth's atmosphere is transparent for these radio frequencies.

Tunka-Rex allows one to make accurate measurements of cosmic ray parameters in the energy range above $100 \mathrm{PeV}$. The developed methods for EAS parameters reconstruction make it possible to reconstruct the energy of the primary particle with an accuracy of $15 \%$ and the depth of the shower maximum with an accuracy of $40 \mathrm{~g} / \mathrm{cm}^{2}$. These methods are based on the analysis of the lateral distribution function, which describes the dependence of the radio signals amplitudes of the antennas from the distance to the shower axis. The distribution of the mean depth of the shower maxima as a function of the primary energy reconstructed by Tunka-Rex and the ones reconstructed by another experiments applied different EAS detection techniques is presented in Fig. 6 [15].

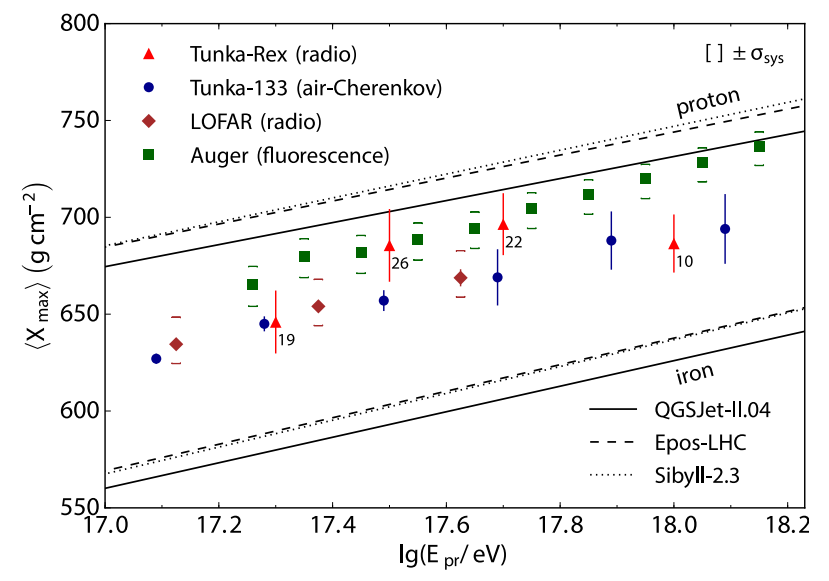

Figure 6. Mean atmospheric depth of shower maximum as a function of energy reconstructed by Tunka-Rex in comparison with other experiments.

\section{Conclusions}

Our immediate goal is to construct the first stage of the TAIGA gamma observatory before the end of 2019; it will include 110-120 wide-angle Cherenkov detectors on an area of 1 $\mathrm{km}^{2}$, three IACT, and $250 \mathrm{~m}^{2}$ of muon detectors. The expected integral sensitivity of this complex for detecting gamma rays with an energy of $100 \mathrm{TeV}$, given the 300-hour observation of a source, will reach approximately $2.5 \times 10^{-13} \mathrm{TeV} \mathrm{cm}^{2} \mathrm{~s}^{-1}$ (Fig. 7), which is higher than the sensitivity of the currently operated arrays for detection of high energy gamma rays. First of all, this complex will allows one to prove experimental potential and advantage of the joint operation of the timing and imaging Cherenkov arrays. Then we intend to construct full scale gamma observatory TAIGA with about 1000 timing Cherenkov detectors and 15 imaging telescopes distributed on area of $10 \mathrm{~km}^{2}$ about. At the same time one square kilometer pilot TAIGA setup will allow one to study: (1) the high-energy end of the spectrum of the brightest galactic gamma ray sources to search for galactic PeVatrons; (2) the high-energy range in the spectrum of bright extragalactic sources (Mkr421 and some other); (3) the mass composition of the cosmic rays in the "ankle" region $\left(10^{14}-10^{16} \mathrm{eV}\right)$ with high statistic and 
accuracy; (4) the cosmic ray anisotropy in the energy range of 100-3000 TeV, etc. As the TAIGA will be the northernmost gamma observatory, its location provides some advantages for observing the sources with large declinations. For example, the gamma ray source in the Tycho Brahe supernova remnant, being almost inaccessible for the HAWC and LHAASO arrays, will remain within the field of view of the TAIGA detectors for 300 hours per year.

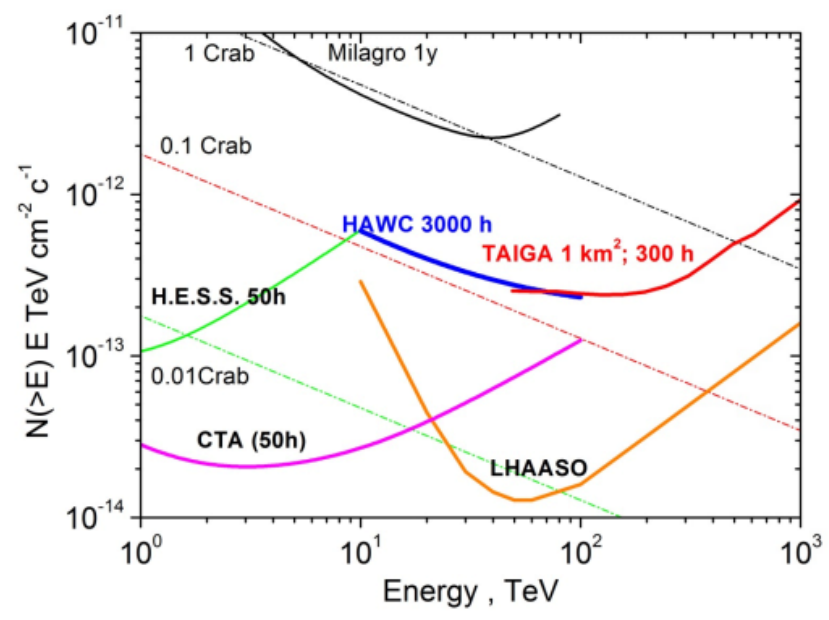

Figure 7. The sensitivity (after $300 \mathrm{~h}$ ) of the $1 \mathrm{~km}^{2}$ TAIGA gamma observatory setup.

Acknowledgements This work is supported by the Russian Federation Ministry of Education and Science (Tunka shared core facilities, unique identifier RFMEFI59317X0005, agreements: $3.9678 .2017 / 8.9$, 3.904.2017/4.6, 3.6787.2017/7.8, 3.6790.2017/7.8), the Russian Foundation for Basic Research (grants 16-02-00738, 17-02-00905, 18-32-00460)

\section{References}

[1] M. Tluczykont, D. Hampf, et al, Astropart. Phys., 56 (2014).

[2] N. Budnev, D. Chernov, et al, Astropart. Phys., 18 (2013).

[3] B. A. Antokhonov, D. Besson, et al, Nucl. Instrum. Methods, 639, 42 (2011).

[4] P. A. Bezyazeekov, N. M. Budnev, et al, JCAP, 1601 (01), 052 (2016).

[5] R. D. Monkhoev, N. M. Budnev, et al, JINST 12 (06), C06019 (2017).

[6] O. Gress, I. Astapov, et al, Nucl. Instrum. Methods, 845, 367 (2017).

[7] A. Porelli, D. Bogorodskii, et al, J. Phys. Conf. Ser. 632, 012041 (2015).

[8] S. F. Berezhnev, N. M. Budnev, et al, Bull. Russ. Acad. Sci. Phys. 79, 344 (2015).

[9] S. F. Berezhnev, D. Besson, et al, Nucl. Instrum. Methods, 692, 98 (2012).

[10] S. F. Berezhnev, N. M. Budnev, et al, Bull. Russ. Acad. Sci. Phys. 79, 348 (2015).

[11] A. Porelli, R. Wischnewski, et al, 35th Int. Cosmic Ray Conf., ICRC2017, Bexco, Busan, Korea, 2017.

[12] V. Kornilov, V. Lipunov, et al, Exper. Astron. 33, 173 (2012).

[13] A. D. Panov, J. H. Adams Jr., et al, Bull. Russ. Acad. Sci.: Phys. 73, 564-567 (2009).

[14] E. Atkin, V. Bulatov, et al, JCAP 7, 20 (2017).

[15] P. Bezyazeekov, N. Budnev et al, Phys.Rev. D97 no.12, 122004 (2018). 